Running Head: Discrimination and Psychiatric Disorders

\title{
Discrimination and Psychiatric Disorders Among Older African Americans
}

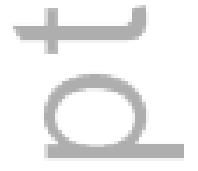

$=$

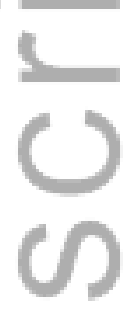

Dawne M. Mouzon, Ph.D. (corresponding author)

Edward J. Bloustein School of Planning \& Public Policy Institute for Health, Health Care Policy, and Aging Research

Rutgers, The State University of New Jersey

33 Livingston Avenue

New Brunswick, NJ 08901

dawne.mouzon@ rutgers.edu; (848) 932-2969

Robert Joseph Taylor, Ph.D. ${ }^{1,2}$

Verna M. Keith, Ph.D. ${ }^{3}$

Emily J. Nicklett, Ph.D. ${ }^{1}$

Linda M. Chatters, Ph.D. ${ }^{1,2,4}$

${ }^{1}$ School of Social Work, University of Michigan, Ann Arbor

${ }^{2}$ Institute for Social Research, Program for Research on Black Americans, University of Michigan, Ann Arbor

${ }^{3}$ Department of Sociology, Race and Ethnic Studies Institute, Texas A \& M University

${ }^{4}$ School of Public Health, University of Michigan, Ann Arbor

Funding/Support:

The data collection for this study was supported by the National Institute of Mental Health (NIMH; U01MH57716), with supplemental support from the Office of Behavioral and Social Science Research at the National Institutes of Health $(\mathrm{NIH})$ and the University of Michigan. The preparation of this manuscript was supported by a grant from the National Institute on Aging to RJT (P30AG1528).

Keywords: Depression, Racial Discrimination, Mood Disorders, Anxiety Disorders Authors have no conflicts of interest.

Word Count: 3,370

$\underline{\text { Key Points: }}$

1. Older African Americans have endured a lifetime of discriminatory practices.

2. Few studies examine the association between everyday discrimination and mental health among older African Americans.

3. Everyday discrimination (whether racial, non-racial, or overall) is associated with higher risk of This is the author manuscript accepted for publication and has undergone full peer review but has not been through the copyediting, typesetting, pagination and proofreading process, which may lead to differences between this version and the Version of Record. Please cite this article as doi: $10.1002 /$ gps.4454 


\section{Running Head: Discrimination and Psychiatric Disorders}

psychiatric disorders, depressive symptoms, and serious psychological distress among African Americans aged 55 and older.

4. This study contributes to a very small but emerging literature on psychiatric disorders among older African Americans.

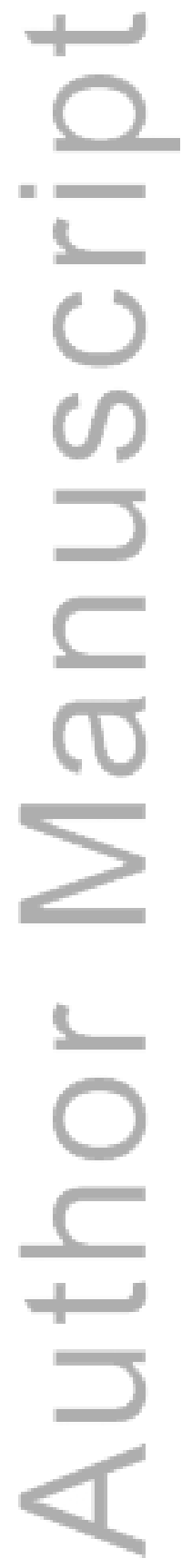




\begin{abstract}
Objectives: This study examined the impact of everyday discrimination (both racial and nonracial) on the mental health of older African Americans.

Methods: This analysis is based on the older African American subsample of the National Survey of American Life (NSAL) ( $\mathrm{n}=773)$. We examined the associations between everyday discrimination and both general distress and psychiatric disorders as measured by the Diagnostic and Statistical Manual of Mental Disorders (DSM-IV). Six dependent variables were examined: lifetime mood disorders, lifetime anxiety disorders, any lifetime disorder, number of lifetime disorders, depressive symptoms as measured by the 12-item Center for Epidemiological Scale of Depression (CES-D), and serious psychological distress as measured by the Kessler 6 (K6).
\end{abstract}

Results: Overall, racial and non-racial everyday discrimination were consistently associated with worse mental health for older African Americans. Older African Americans who experienced higher levels of overall everyday discrimination had higher odds of any psychiatric disorder, any lifetime mood disorder, any lifetime anxiety disorder, the number of lifetime DSM-IV disorders, in addition to elevated levels of depressive symptoms and serious psychological distress. These findings were similar for both racial discrimination and non-racial discrimination.

Conclusions: This study documents the harmful association of not only racial discrimination, but also non-racial (and overall) discrimination with the mental health of older African Americans. Specifically, discrimination is negatively associated with mood and anxiety disorders as well as depressive symptoms and psychological distress. 


\section{INTRODUCTION}

Over the past 10 years, there has been a dramatic increase in psychiatric research on the prevalence of mental disorders among older African Americans (Aranda et al., 2012; Chatters et al., 2015; Ford et al., 2007; Jimenez et al., 2010; Woodward et al., 2012; Woodward et al., 2013). Nonetheless, there remains a paucity of in-depth work investigating critical risk and protective factors for psychiatric disorders and mental health within this population. Everyday discrimination is a critical risk factor beginning to garner a significant amount of interest. The goal of this study is to investigate the impact of racial and non-racial everyday discrimination on psychiatric disorders and mental health among older African Americans.

Over the course of their lives, older African Americans have experienced major discrimination in the form of segregated neighborhoods, political disenfranchisement, underresourced schools, and employment discrimination. Major discrimination is defined as occurrences that involve being denied or severely limited in access to opportunities in important life domains such as work, housing, and education (Williams, Yu and Jackson, 1997). Some examples of major discrimination include having been unfairly fired or denied a promotion; not hired for a job; prevented from buying, renting, or leasing a home or apartment; denied a bank loan; and denied access to educational opportunities. Older African Americans have also been subjected to high levels of everyday discrimination. Everyday discrimination is defined as chronic, and unfair treatment that occurs in commonplace social encounters (Kessler et al., 1999) and includes receiving poor restaurant service, being perceived as not smart or dishonest, being 
Running Head: Discrimination and Psychiatric Disorders

treated with less respect, and being followed in stores. African Americans experience higher prevalence of everyday discrimination than do non-Hispanic Whites (Ayalon and Gum, 2011; Beatty Moody et al., 2014; Luo et al., 2012; Turner and Avison, 2003; Williams et al., 1997). Further, both African American adults (Kessler et al., 1999) and African American adolescents (Seaton et al., 2010) report that race/ethnicity is the most prevalent attribution for discriminatory acts. Of particular importance, both major life discrimination and everyday discrimination are associated with negative mental health outcomes for African Americans (Lewis et al., 2015; Williams and Mohammed, 2013), a position formally acknowledged by the American Psychiatric Association (American Psychiatric Association, 2006).

Relatively little is known about the impact of everyday discrimination on psychiatric disorders among older African Americans. In this study, we examine the association between everyday discrimination (racial, non-racial, and overall) and mental health among African Americans aged 55 and older. These respondents were born between 1909 and 1947, with most clustering around 1935. This cohort was born during the period directly preceding the Civil Rights Movement of the 1960s and was among the last to endure the traumatic and overtly racialized experiences endemic to the Jim Crow system of legalized discrimination. Exploring this important historical cohort can help us better understand the possible mental health impacts of the protracted experience of both legalized and extralegal discrimination over their life course. For example, a cohort that has survived these experiences may have developed effective coping mechanisms that mitigate the impact of discrimination on their mental health. Conversely, the 
Running Head: Discrimination and Psychiatric Disorders

cumulative burden of discriminatory experiences may predispose members of this cohort to worse mental health status.

One study found more depressive symptoms among older African Americans who reported high levels of everyday discrimination (Marshall and Rue, 2012). To the best of our knowledge, this the first study to investigate the impact of everyday discrimination on psychiatric disorders among older African Americans. We add to the present literature in several critical ways. First, we examine six indicators of mental health and psychiatric disorders. We also explore multiple outcomes spanning lifetime mental disorders, 30-day general distress, and depressive symptoms. Second, we control for chronic health conditions given their strong association with mental health (Katz, 1996). Third, we investigate the associations of overall, racial, and non-racial everyday discrimination on mental health, an initiative that has been noted in recent research as one of the next steps in this field (Lewis et al., 2015).

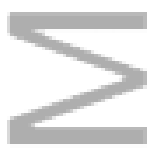

\section{METHODS}

\section{Sample}

The National Survey of American Life: Coping with Stress in the 21st Century (NSAL) is a cross-sectional study conducted by the Program for Research on Black Americans at the University of Michigan's Institute for Social Research between 2001 and 2003. A total of 6,082 face-to-face interviews were conducted with persons aged 18 or older, including 3,570 African Americans, 891 non-Hispanic whites, and 1,621 Blacks of Caribbean descent. The NSAL included 837 African Americans aged 55 years or older; after accounting for missing data, the 
Running Head: Discrimination and Psychiatric Disorders

analytic sample is comprised of 773 respondents. The overall response rate for the NSAL was $72.3 \%$ and respondents were compensated for their time. Design and sample characteristics of the NSAL are described in more detail elsewhere (Heeringa et al., 2004; Jackson et al., 2004; Pennell et al., 2004). This study was approved by the University of Michigan's Institutional Review Board.

\section{Measures}

The following six dependent variables are utilized: 1) lifetime mood disorders (major depressive disorder, dysthymia, and bipolar I \& II disorders); 2) lifetime anxiety disorders (panic disorder, agoraphobia, social phobia, generalized anxiety disorder, obsessive compulsive disorder, and posttraumatic stress disorder); 3) any lifetime disorder; 4) number of lifetime disorders; 5) depressive symptoms as measured by the CES-D; and 6) serious psychological distress as measured by the Kessler 6 (K6). The DSM-IV World Mental Health Composite International Diagnostic Interview (WMH-CIDI), a fully structured diagnostic interview, was used to assess mental disorders (Kessler and Ustun, 2004). Obsessive-compulsive disorder was assessed using the CIDI-Short Forms (Kessler et al., 1998). Any lifetime disorder includes mood and anxiety disorders, as well as drug abuse, drug dependence, alcohol abuse, alcohol dependence, oppositional defiant disorder, conduct disorder, attention deficit/hyperactivity disorder, separation anxiety, anorexia, bulimia, and binge eating disorder. The number of disorders is a count of all previously mentioned disorders.

Depressive symptoms were assessed using the 12-item version of the Center for Epidemiological Studies-Depression scale (CES-D) (Radloff, 1977). This abbreviated CES-D 
Running Head: Discrimination and Psychiatric Disorders

has acceptable reliability and a similar factor structure compared to the original version. Item responses are coded from 0 ("rarely or none of the time") to 3 ("most or all of the time") and focus on experiences in the past 30 days. These 12 items measure the extent to which respondents: had trouble keeping their mind on tasks, enjoyed life, had crying spells, could not get going, felt depressed, felt hopeful, felt restless, felt happy, felt just as good as other people, felt that everything was an effort, felt that people were unfriendly, and felt that people dislike them. Positive valence items were reverse-coded and summed into a continuous measure ranging from 0/low through 36/high $(\mathrm{M}=5.14, \mathrm{SD}=4.48)(\alpha=0.77)$. Research on the psychometric properties of the CES-D on older African Americans finds adequate reliability with good internal consistency (Long Foley et al., 2002).

Serious psychological distress (SPD) was measured by the K6, a six-item scale designed to assess non-specific psychological distress including symptoms of depression and anxiety in the past 30 days (Kessler et al., 2002; Kessler et al., 2003). The K6 includes items designed to identify individuals with a high likelihood of having a diagnosable mental illness and associated limitations. More specifically, the K6 is intended to identify persons with mental health problems severe enough to cause moderate to serious impairment in social and occupational functioning and to require treatment. Each item was measured on a 5-point Likert scale ranging from 0 (none of the time) to 4 (all of the time). Positive valence items were reverse-coded and summed with higher scores reflecting higher levels of psychological distress $(\alpha=0.83)$. The K6 has consistent psychometric properties across major socio-demographic sub-samples (Kessler et al., 2002); 
Running Head: Discrimination and Psychiatric Disorders

however, there are very few measurement studies or other research using the K6 among older African Americans.

\section{Independent Variables}

The measure of everyday discrimination was designed to assess interpersonal forms of routine experiences of unfair treatment (Williams et al., 1997). Ten items were used to measure everyday discrimination in the NSAL, including the frequency with which one: is treated with less courtesy; is treated with less respect; received poor restaurant service; is perceived as not smart; is perceived as dishonest; is perceived as not as good as others; is feared, and is insulted, harassed, and followed in stores. Response values for each item were: 5 (almost every day), 4 (at least once a week), 3 (a few times a month), 2 (a few times a year), 1 (less than once a year), and 0 (never). These 10 items were combined to create a summary scale, with higher scores on this summary scale indicating higher levels of discrimination $(\alpha=0.90)$. For each item of everyday discrimination, respondents were first asked how often this type of discrimination occurred (e.g., receive poor service in a store or restaurant) and following this they were asked to identify the primary reason for such experiences (e.g., race, gender, age, skin color, height or weight). Using this series of questions, we created three summary scales of everyday discrimination. One variable is a summary variable of overall everyday discrimination (regardless of perceived attribution). The remaining two variables were created to reflect both perceived discrimination that was specifically attributed to race and perceived discrimination that was not attributed to race. Consequently, in this analysis we assess three everyday discrimination measures - overall 
Running Head: Discrimination and Psychiatric Disorders

everyday discrimination (regardless of perceived attribution), everyday racial discrimination, and everyday non-racial discrimination.

As noted earlier, for each indicator of everyday discrimination (e.g., followed in stores) respondents were asked to identify the primary reason for such experiences (e.g., race, gender, age). Each of the three summary variables has values that range from $0-50$. A value of 50 on the summary variable of overall discrimination would indicate that a person reported being discriminated almost everyday (which has a value of 5) on all 10 indicators of everyday discrimination ( $5 \times 10$ indicators). A value of 0 on the summary variable of overall discrimination would indicate that a person reported never being discriminated on any of the 10 indicators of discrimination. A value of 50 on the summary variable of discrimination due to race would indicate that a person reported being discriminated against due to their race almost everyday on all 10 indicators of everyday discrimination.

Sociodemographic factors (e.g., age, gender, family income, education, and marital status) are utilized as control variables. Age and education were coded in years and family income was coded in dollars. Marital status was coded into two categories: 1) married and cohabiting and 2) separated, divorced, widowed, and never married. Physical health, measured by respondents' reports of doctor-diagnosed chronic physical health conditions, was based on a count of 11 potential conditions.

\section{Analysis Strategy}

The percentages represent weighted proportions based on the distribution of African Americans in the population. Multivariate logistic regression analysis was used to examine the 
Running Head: Discrimination and Psychiatric Disorders

association of discrimination with the prevalence of any mood, any anxiety, and any lifetime disorder. Odds ratio estimates and $95 \%$ confidence intervals are presented. Poisson and negative binomial regressions were used to model the number of lifetime disorders, depressive symptoms and serious psychological distress. In particular, univariate measures (i.e., means, variance, histogram) of number of disorders and depressive symptoms revealed a Poisson distribution. For these dependent variables, the mean was approximately equal to the variance within older African Americans, satisfying the main assumption of Poisson regression. In contrast, serious psychological distress displayed overdispersion, as the variance exceeded the mean in this subsample of older African Americans. In this case, negative binomial regression, a generalization of the Poisson model, was more appropriate. For the Poisson and negative binomial regressions, rate ratio estimates and $95 \%$ confidence intervals are presented. In all regressions, a design-corrected $F$ statistic is presented. The standard errors reflect the recalculation of variance using the study's complex design. All analyses were conducted using SAS 9.2, which uses the Taylor expansion approximation technique for calculating the complex design-based estimates of variance.

1

Pocic

Socio-demographic characteristics of the older African American sample are presented in Table 1. Age of respondents ranged from 55 to 93 years $(M=66.6 ; \mathrm{SD}=7.26)$. Approximately $60 \%$ of the respondents are women and four out of ten $(39.9 \%)$ are married. The average imputed family income is $\$ 32,853(\mathrm{SD}=\$ 32,730)$ and the average years of education is 11.5 $(\mathrm{SD}=2.97)$. Lastly, respondents average 2.58 chronic health problems. Older African Americans 
Running Head: Discrimination and Psychiatric Disorders

reported a moderate degree of mental health problems. Just under $8 \%$ of the sample met criteria for any mood disorder and more than $13 \%$ met criteria for any anxiety disorder. Roughly a quarter $(27.2 \%)$ of respondents met criteria for any lifetime DSM disorder. The average score on the depressive symptoms scale was 5.16 (range: 0-33) and the average score on the serious psychological distress scale was 2.65 (range: 0-23).

= - Respondents reported numerous instances of discrimination. In terms of overall patterns, roughly $10 \%$ of respondents reported that "a few times a month," people acted as if they were better than them, people acted as if they were not smart, and they were treated with less courtesy than others. Roughly 2 out of 5 respondents report that they experience "people acting like they are better than them" at least a few times a year. Although not as frequent, many older African Americans indicated that at least "a few times a year" they had the experience of being followed around in stores (20\%), received poor service in stores and restaurants (26\%), people acted as if they were afraid of them (15\%), and people treated them as if they were dishonest (18\%). Older African American men were significantly more likely to report experiencing everyday discrimination than their female counterparts and respondents aged 55-64 reported experiencing discrimination more frequently than respondents aged 70 and over. Additionally, the majority of respondents attributed their experiences with discrimination to their race.

\section{Table 1 about here}

Table 2 presents results from the multivariate regression analysis of everyday discrimination on the six mental health dependent variables. The top panel of the table presents odds ratios and risk ratios based on the overall everyday discrimination analysis, the middle 
Running Head: Discrimination and Psychiatric Disorders

panel presents results regarding everyday racial discrimination, and the bottom panel displays results regarding everyday non-racial discrimination. All models control for age, gender, income, education, marital status, and number of chronic health problems.

\section{Table 2 about here}

Overall everyday discrimination (discrimination that is attributed to any cause, not merely race) was significantly and positively associated with all six mental health outcomes. Older African Americans who reported higher overall everyday discrimination had significantly higher odds of meeting criteria for any lifetime mood disorder, any lifetime anxiety disorder, any lifetime disorder, number of DSM-IV mental disorders, and higher levels of both depressive symptoms and psychological distress. Almost identical results were found for everyday racial discrimination; older African Americans who reported higher levels of everyday racial discrimination had worse mental health outcomes on five of the six measures examined. The analysis for any anxiety disorder bordered significance $(\mathrm{p}=0.052)$. Table 2 also reports the analysis of the summary of the six measures of mental health on everyday non-racial discrimination. For five of the six measures, having high levels of perceived non-racial based discrimination was associated with poorer mental health. The one exception was that lifetime mood disorder was not significantly associated with non-racial discrimination.

\section{DISCUSSION}

In this study, we examined the association between three measures of everyday discrimination (overall discrimination, racial discrimination, and non-racial discrimination) and mental health among older African Americans aged 55 and older. This cohort has endured 
Running Head: Discrimination and Psychiatric Disorders

lengthy periods of discrimination over their life course yet little research has specifically investigated the impact of these experiences for later life. Regardless of its nature, whether overall, racial, or of a non-racial nature, everyday discrimination was consistently associated with worse mental health for African Americans over age 55. In terms of psychiatric disorders, older African Americans who experienced higher levels of overall everyday discrimination had highēr odds of any lifetime mood disorder, any lifetime anxiety disorder, and number of lifetime DSM-IV disorders. Relative to older adults who perceived less overall everyday discrimination, those with higher levels of overall everyday discrimination also had elevated levels of depressive symptoms and serious psychological distress. These findings were very similar in both magnitude and statistical significance for everyday racial discrimination; older African Americans with higher levels of general everyday discrimination also had worse mental health across five of six outcomes. Our findings also indicated that - with the exception of lifetime mood disorder - non-racial discrimination was associated with worse mental health among older African Americans.

Our findings are consistent with studies investigating the role of everyday discrimination on psychiatric disorders. For example, one study found that everyday discrimination particularly the frequency with which respondents were treated as less smart, dishonest, threatened or called names - increased the odds of panic attacks in the past 12 months (Hearld et al., 2015). Everyday discrimination is also correlated with higher risk of three psychotic experiences (auditory hallucinations, visual hallucinations, and delusional ideations) (Oh et al., 2014) and higher odds of both 12-month substance abuse disorder and lifetime substance abuse 
Running Head: Discrimination and Psychiatric Disorders

(Clark, 2014). Everyday discrimination is also related to higher odds of social anxiety disorder among African Americans (Levine et al., 2014) and is associated with both psychological distress and poor well-being (Williams et al., 1997).

For aging African American adults, we found that a lifetime of everyday discrimination exposure was associated with mental health problems. Everyday discrimination may exert adverse psychological effects via multiple pathways (e.g., subconscious internalization of negative racialized stereotypes, increased vigilance during common social encounters). As an ongoing source of unfair treatment, everyday discrimination is considered a chronic stressor of a persistent nature (Williams and Mohammed, 2009). Much like the pervasive effects of material hardships (e.g., enduring poverty, nutritional deficits), the chronic nature of everyday discrimination represents a persistent challenge to the emotional well-being of African Americans. Although clinicians may be primed to notice and respond to major lifetime events affecting aging patients (e.g., divorce, widowhood, major health events), the chronically stressful nature of everyday discrimination is also a vital consideration for clinical practice involving older African Americans.

As with any study, this analysis has important limitations to consider. First, the National Survey of American Life is cross-sectional; therefore, it is not possible to ascertain causality for the association between everyday discrimination and mental health outcomes. That is, African Americans with mental health concerns could be more likely to report experiencing everyday discrimination. However, longitudinal analyses have largely found that discrimination precedes mental health/illness, not the reverse (Brown et al., 2000). Second, the number of cases for 
Running Head: Discrimination and Psychiatric Disorders

several lifetime disorders is low and, consequently, we are unable to conduct disorder-specific analyses. Third, it would have been preferable to examine 12-month as opposed to lifetime disorders to lessen the temporal differences between the dependent and discrimination variables. However, the number of cases of 12-month disorders was too few to conduct reliable analysis.

Future research would benefit from the use of longitudinal data to more definitely establish the temporal nature of racial discrimination and mental health. Although existing longitudinal research supports the assumption that racial discrimination precedes health outcomes (i.e., Barnes et al., 2008; Brown et al., 2000; Schulz, 2006), this relationship should be examined using more recent data from nationally representative samples. Another vital direction for future research involves the number and types of perceived attributions. Research shows that individuals who report more than one perceived reason for a discriminatory event exhibit higher risk for both depressive symptoms and lifetime major depression (Gayman and Barragan, 2013; Grollman, 2014). The intersections of different discrimination attributions are also important. For example, examining the gendered nature of racial discrimination (Harnois and Ifatunji, 2010) and other intersections (e.g., race/ethnicity and age) are crucial considerations for future research.

In conclusion, the present study has four important advantages. First, these data were derived from the 2001-2003 National Survey of American Life, which includes the largest nationally representative dataset of African Americans in the United States. These data allowed for the-application of important demographic and health covariates and produced findings that are generalizable to the older African American population. Second, whereas previous studies of 
Running Head: Discrimination and Psychiatric Disorders

everyday discrimination investigate its impact on only a single measure, this study focused on several indicators of mental illness and psychiatric disorders. Third, given important psychometric research showing that mental illness, mental health, and psychological distress are non-overlapping concepts (Payton, 2009), the consistency of our findings across six distinct measures of mental health and disorder is notable in demonstrating the impact of both general everȳday and specifically racial everyday discrimination. Finally, our findings are also robust across different time frames for symptoms such as within the past 30 days and anytime during life course. Taken together, these findings demonstrate that discrimination continues to have a negative impact on the everyday lives of aging African Americans. 
Running Head: Discrimination and Psychiatric Disorders

\section{REFERENCES}

American Psychiatric Association. 2006. Resolution Against Racism and Racial Discrimination and Their Adverse Impacts on Mental Health. American Psychiatric Association: Arlington, VA.

Aranda MP, Chae DH, Lincoln KD, et al. 2012. Demographic correlates of DSM-IV major depressive disorder among older African Americans, Black Caribbeans, and nonHispanic Whites: results from the National Survey of American Life. Int J Geriatr Psychiatry 27: 940-947.

Ayalon L, Gum AM. 2011. The relationships between major lifetime discrimination, everyday discrimination, and mental health in three racial and ethnic groups of older adults. Aging Ment Health 15: 587-594.

Beatty DL, Matthews KA, Bromberger JT, et al. 2014. Everyday discrimination prospectively predicts inflammation across 7-years in racially diverse midlife women: study of women's health across the nation. J Soc Issues 70: 298-314.

Brown TN, Williams DR, Jackson JS, et al. 2000 'Being black and feeling blue': the mental health consequences of racial discrimination. Race Soc 2: 117-131.

Chatters LM, Taylor RJ, Woodward AT, et al. 2015. Social support from church and family members and depressive symptoms among older African Americans. Am J Geriatr Psychiatry 23: 559-567.

Clark TT. 2014. Perceived discrimination, depressive symptoms, and substance use in young adulthood. Addict Behav 39: 1021-1025.

Ford BC, Bullard KM, Taylor RJ, et al. 2007. Lifetime and 12-month prevalence of Diagnostic and Statistical Manual of Mental Disorders, fourth edition disorders among older African Americans: findings from the National Survey of American Life. Am J Geriatr Psychiatry 15: 652-659.

Gayman MD, Barragan J. 2013. Multiple perceived reasons for major discrimination and

depression. Soc Ment Health 3: 203-220.

Grollman, E.A. 2012. Multiple forms of perceived discrimination and health among adolescents and young adults. J Health Soc Behav 53: 199-214.

Grollman, E.A. 2014. Multiple disadvantaged statuses and health: the roles of multiple forms of discrimination. J Health Soc Behav 55: 3-19. 
Running Head: Discrimination and Psychiatric Disorders

Harnois, C.E., \& Ifatunji, M. 2011. Gendered measures, gendered models: toward an interpersonal analysis of interpersonal discrimination. Ethn Racial Stud 34: 1006-1028.

Hearld KR, Budhwani H, Chavez-Yenter D. 2015. Panic attacks in minority Americans: the effects of alcohol abuse, tobacco smoking, and discrimination. J Affect Disord 174: 106112.

Heeringa SG, Wagner J, Torres M, et al. 2004. Sample designs and sampling methods for the Collaborative Psychiatric Epidemiology Studies (CPES). Int J Methods in Psychiatr Res 13: $221-240$.

Jackson JS, Torres M, Caldwell CH, et al. 2004. The National Survey of American Life: a study of racial, ethnic and cultural influences on mental disorders and mental health. Int $J$ Methods in Psychiatr Res 13: 196-207.

Jimenez DE, Alegría M, Chen C, et al. 2010. Prevalence of psychiatric illnesses among ethnic minority elderly. J Am Geriatr Soc 58: 256-264.

Katz IR. 1996. On the inseparability of mental and physical health in aged persons: lessons from depression and medical comorbidity. Am J Geriatr Psychiatry 4: 1-16.

Kessler RC, Ustün TB. 2004. The World Mental Health (WMH) survey initiative version of the World Health Organization (WHO) Composite International Diagnostic Interview (CIDI). Int J Methods in Psychiatr Res 13: 93-121.

Kessler RC, Andrews G, Mroczek D, et al. (1998) The World Health Organization Composite International Diagnostic Interview short-form (CIDI-SF). Int J Methods in Psychiatr Res 7: $171-185$.

Kessler RC, Mickelson KD, Williams DR. 1999. The prevalence, distribution, and mental health correlates of perceived discrimination in the United States. J Health Soc Behav 40: 208230.

Kessler RC, Andrews G, Colpe LJ, et al. 2002. Short screening scales to monitor population prevalences and trends in non-specific psychological distress. Psychol Med 32: 959-976.

Kessler RC, Barker PR, Colpe LJ, et al. 2003. Screening for serious mental illness in the general population. Arch Gen Psychiatry 60: 184-189.

Levine DS, Himle JA, Abelson JM, et al. 2014. Discrimination and social anxiety disorder among African-Americans, Caribbean blacks, and non-Hispanic whites. J Nerv Ment Dis 202: $224-230$. 
Running Head: Discrimination and Psychiatric Disorders

Lewis TT, Cogburn CD, Williams DR. 2015. Self-reported experiences of discrimination and health: scientific advances, ongoing controversies, and emerging issues. Annu Rev Clin Psychol 11: 407-440.

Long Foley K, Reed PS, Mutran EJ, et al. 2002. Measurement adequacy of the CES-D among a sample of older African-Americans. Psychiatry Res 109: 61-69.

Luo Y,Xu J, Granberg E, et al. 2012. A longitudinal study of social status, perceived discrimination, and physical and emotional health among older adults. Res Aging 34: 275-301.

Marshall GL, Rue TC. 2012. Perceived discrimination and social networks among older African Americans and Caribbean blacks. Fam Community Health 35: 300-311.

Oh H, Yang LH, Anglin DM, et al. 2014. Perceived discrimination and psychotic experiences across multiple ethnic groups in the United States. Schizophr Res 157: 259-265.

Payton AR. 2009. Mental health, mental illness, and psychological distress: same continuum or distinct phenomena? J Health Soc Behav 50: 213-227.

Pennell B-E, Bowers A, Carr D, et al. 2004. The development and implementation of the National Comorbidity Survey Replication, the National Survey of American Life, and the National Latino and Asian American Survey. Int J Methods Psychiatr Res 13: 241-269.

Radloff LS. 1977. The CES-D scale: a self-report depression scale for research in the general population. Appl Psychol Meas 1: 385-401.

Schulz AJ, Gravlee CG, Williams DR, et al. 2006. Discrimination, symptoms of depression, and self-rated health among African American women in Detroit: results from a longitudinal analysis. Am J Public Health 96: 1265-1270.

Seaton EK, Caldwell CH, Sellers RM, et al. 2010. Developmental characteristics of African American and Caribbean Black adolescents' attributions regarding discrimination. J Res Adolesc 20: 774-788.

Turner RJ, Avison WR. 2003. Status variations in stress exposure: implications for the interpretation of research on race, socioeconomic status, and gender. J Health Soc Behav 44: 488-505.

Williams DR, Mohammed SA. 2009. Discrimination and racial disparities in health: evidence and needed research. J Behav Med 32: 20-47. 
Running Head: Discrimination and Psychiatric Disorders

Williams DR, Mohammed SA. 2013. Racism and health I: pathways and scientific evidence. Am Behav Sci 57: 1152-1173.

Williams DR, Yu Y, Jackson JS, et al. 1997. Racial differences in physical and mental health: socioeconomic status, stress, and discrimination. J Health Psychol 2: 335-351.

Woodward A, Taylor RJ, Bullard K, et al. 2012. Prevalence of lifetime DSM-IV affective

disorders among older African Americans, Black Caribbeans, Latinos, Asians, and NonHispanic White people. Int J Geriatr Psychiatry 27: 816-827.

Woodward AT, Taylor RJ, Abelson JM, et al. 2013. Major depressive disorder among older African Americans, Caribbean blacks, and non-Hispanic whites: secondary analysis of the National Survey of American Life. Depress Anxiety 30: 589-597.

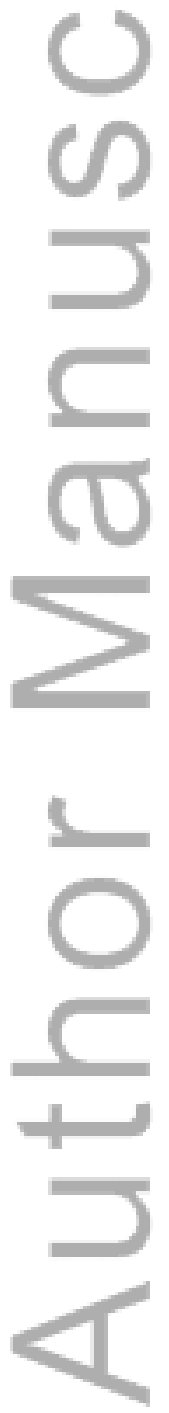

This article is protected by copyright. All rights reserved. 
Running Head: Discrimination and Psychiatric Disorders

Table 1. Demographic Characteristics of the Sample and Distribution of Study Variables, 2001-2003 National Survey of American Life ${ }^{\mathrm{a}}$

\begin{tabular}{|c|c|c|c|c|c|}
\hline & $\%$ & $\mathbf{N}$ & Mean & S.D. & Range \\
\hline Age & & 772 & 66.65 & 7.30 & $55-93$ \\
\hline \multicolumn{6}{|l|}{ Gender } \\
\hline Male & 40.74 & 276 & & & \\
\hline Female & 59.26 & 496 & & & \\
\hline \multicolumn{6}{|l|}{ Marital Status } \\
\hline Married/partnered & 40.40 & 239 & & & \\
\hline Not Married & 59.60. & 533 & & & \\
\hline Income & & 772 & 33306.94 & 33803.62 & $0-450,000$ \\
\hline Education & & 772 & 11.55 & 2.98 & $0-17$ \\
\hline No. of Chronic Health Conditions & & 733 & 2.59 & 1.59 & $0-11$ \\
\hline \multicolumn{6}{|l|}{ Mental Health Conditions } \\
\hline$\overline{\text { Mood Disorder }}$ & 7.36 & 60 & & & \\
\hline Anxiety Disorder & 13.42 & 100 & & & \\
\hline Any Disorder & 27.22 & 198 & & & \\
\hline \# of Disorders & & 733 & 0.45 & 0.73 & $0-3$ \\
\hline \# of Depressive Symptoms (CES-D) & & 730 & 5.16 & 4.50 & $0-33$ \\
\hline Serious Psychological Distress (K-6) & & 732 & 2.65 & 3.16 & $0-23$ \\
\hline \multicolumn{6}{|l|}{ Everyday Discrimination Scales } \\
\hline Overall Everyday Discrimination & & 772 & 8.74 & 7.28 & $0-50$ \\
\hline Everyday Racial Discrimination & & 772 & 5.70 & 7.43 & $0-50$ \\
\hline Everyday Non-Racial Discrimination & & 772 & 1.93 & 4.65 & $0-48$ \\
\hline
\end{tabular}

${ }^{a}$ Data are given as weighted means and weighted standard deviations for continuous variables and unweighted frequencies and weighted percentages for categorical variables.

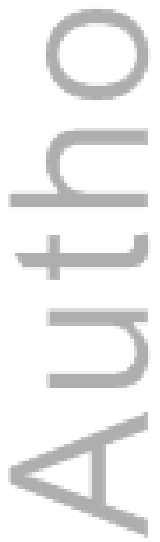

This article is protected by copyright. All rights reserved. 
Table 2. Multivariate Analysis of Overall Everyday Discrimination, Everyday Racial Discrimination, and Everyday Non-Racial Discrimination on Psychiatric Disorders and Distress, 2001-2003 National Survey of American Life

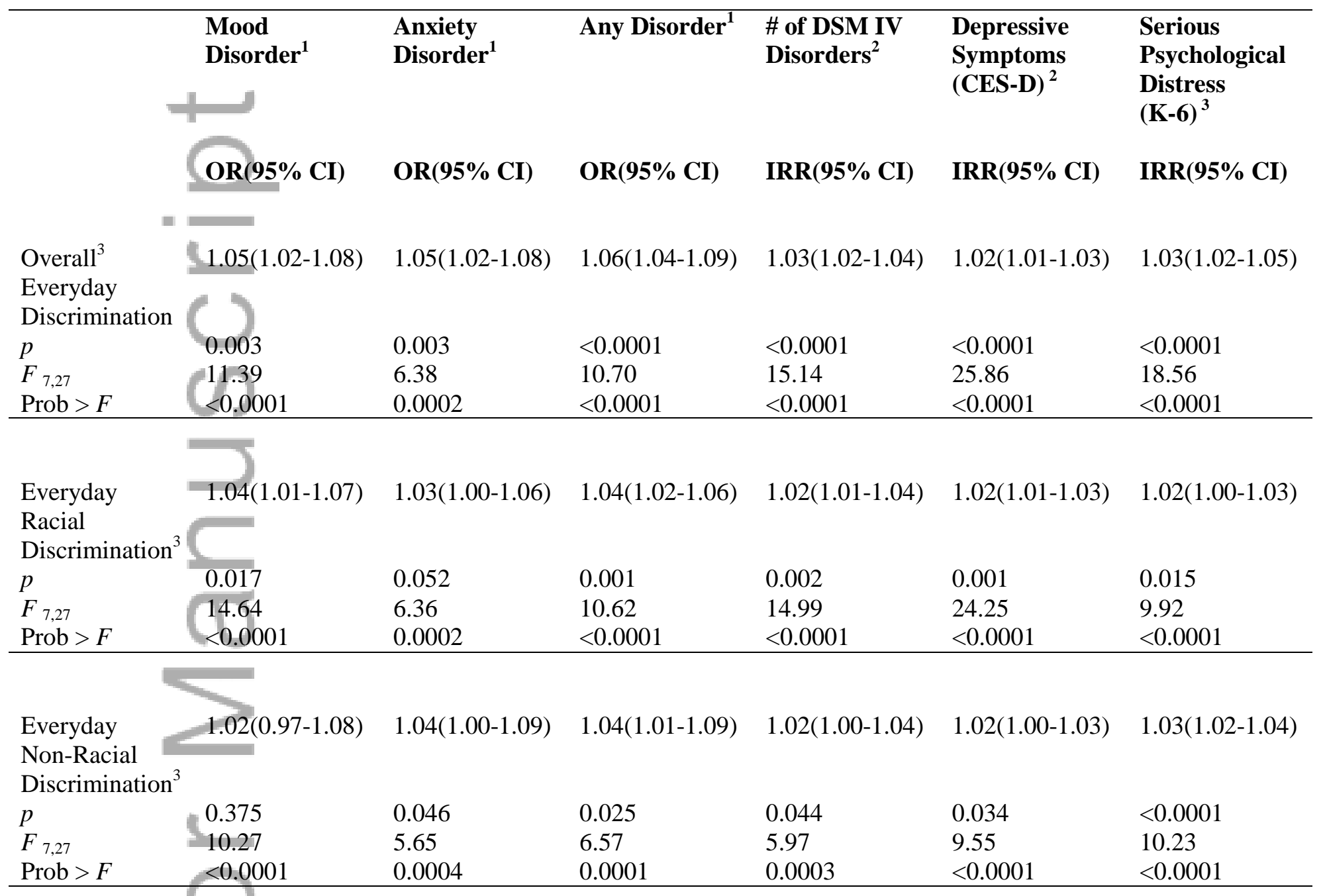

$\mathrm{OR}=$ odds ratio; $\mathrm{IRR}=$ incidence rate ratio; $95 \% \mathrm{CI}=95 \%$ confidence interval.

Note: Significance test of the individual parameter estimates were based on a complex design-corrected $t$-test.

${ }^{1}$ Multivariate logistic regression OR $(95 \% \mathrm{CI})$ and $p$-values were reported.

${ }^{2}$ Multivariate Poisson regression IRR $(95 \% \mathrm{CI})$ and $p$-values were reported.

${ }^{3}$ Negative binomial regression IRR $(95 \% \mathrm{CI})$ and $p$-values were reported.

${ }^{4}$ Age, gender, income, education, marital status, and number of chronic health problems were controlled for in the final models. 